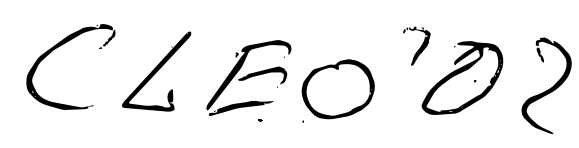

\title{
Maskless Fresnel zone plates for Dense Embedded Optical components
}

\author{
Erica Bricchi, John D. Mills, Peter G. Kazansky and Bruce G. Klappauf \\ Optoelectronics Research Centre, University of Southampton, Southampton SO17 1BJ, UK \\ Jeremy J. Baumberg \\ Department of Physics and Astronomy, University of Southampton. Southampton SOI 7 1BJ, UK
}

\begin{abstract}
We demonstrate - the fabrication of maskless large diameter Fresnel zone plates embedded deep inside silica glass substrates, using direct write techniques employing femtosecond laser pulses. High quality focussing properties are achieved.
\end{abstract}

In the last few years femtosecond lasers have proved to be of great utility for micromachining within the bulk of transparent materials [1,2]. When an intense femtosecond laser pulse is tightly focused into a transparent material, high-order non-linear absorption allows the energy to be deposited predominantly within the focal volume producing a permanent refractive index modification of up to $10^{-2}$ in the bulk of the transparent material. This process allows local patteming inside transparent media into 3D structures, allowing for instance the assembly of many optical components in a single glass block, producing rigid and environmentally tolerant devices.

Using this simple technique of direct writing, we are able to write a maskless Fresnel zone plate (radius of 0.997 $\mathrm{mm}, 158$ Fresnel zones) embedded in silica glass. Femtosecond laser pulses (pulse duration $120 \mathrm{fs}$, wavelength 850 $\mathrm{nm}$, repetition rate $250 \mathrm{kHz})$ are focussed into the glass using a microscope objective $(50 \times, \mathrm{NA}=0.55)$ and the glass element is translated with a three-dimensional, computer controlled stage of $100 \mathrm{~nm}$ positional accuracy in all directions. For the experiments we use an average power of $250 \mathrm{~mW}$, pulse energies up to $1 \mu \mathrm{J}$ and a scan speed of $400 \mu \mathrm{m} / \mathrm{s}$. In order to characterize the lens we illuminate the sample with a white light source from below, and viewed the lens and its focal plane with a microscope ( $4 \times$ objective, $\mathrm{NA}=0.1$ ). Focussing on the directly-written lens plane, the altemating black and white Fresnel zones of the written lens are clearly visible (Fig.1).

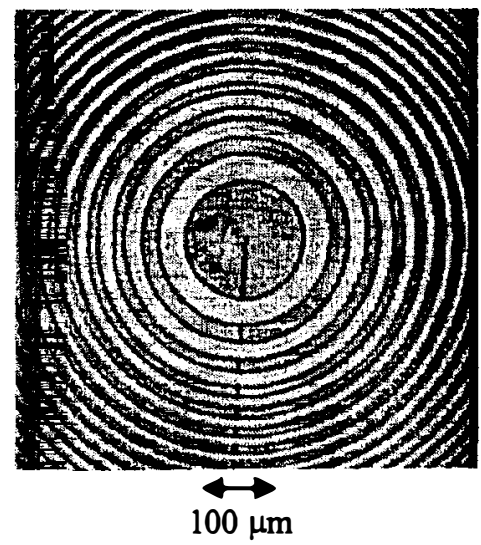

Fig. 1. Optical microscope image of the Fresnel zone plate. 


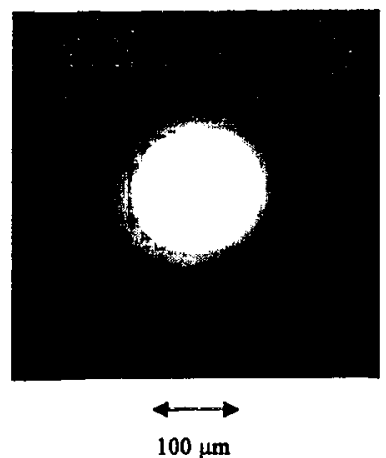

Fig. 2. Optical microscope image of the primary focus of the Fresnel zone plate.

Moving above the directly-written plane of the Fresnel lens, we find the position of the primary focus of the lens to be about $1 \mathrm{~cm}$ from the lens as expected (Fig. 2). The size of the focal spot is $100 \mu \mathrm{m}$, in agreement with the structure of the Fresnel zone plate, while the chromatic aberration leads to the expected focal separation of colours along the optical axis. As predicted by theory, two secondary foci are also seen. These observations prove the feasibility of directly writing complex interferometric components into the body of glass substrates, and show that it is feasible to combine them into devices.

In conclusion, we demonstrate the ability to directly write Fresnel zone plates using a simple maskless technique instead of the typical complex fabrication processes involving electron-beam [3] and $x$-ray lithography [4]. This technique allows us to embed the lens in the glass allowing the possibility of writing multiple lens systems in three dimensions. We are currently investigating the possibility of writing Fresnel zone plates on the cleaved face of optical fibres for which preliminary results prove encouraging.

1. C. B. Shaffer, A. Brodeur, J. F. Garcia, and E. Mazur, "Micromachining bulk glass by use of femtosecond laser pulses with nanojoule energy," Opt. Lett. 26, 93-95 (2001).

2. K. Miura, J. Qiu, H. Inouye, T. Mitsuyu, and K. Hirao "Photowritten optical waveguides in various glasses with ultashort pulse laser," Appl. Phys. Lett. 71, 3329-3331 (1997).

3. T. Erdogan, O. King, G. W. Wicks, D. G. Hall, E. H. Anderson, and M. J. Rooks, "Circulary symmetric operation of a concentric-circlegrating, surface-emitting, AlGaAs/GaAs quantum-well semiconductor laser" Appl. Phys. Lett. 60, 1921-1923 (1992).

4. A. Ozawa, T. Tamamura, T. Ishii, H. Yoshihara, and T. Kagoshima, "Application of X-ray mask fabrication technologies to high resolution, large diameter Ta Fresnel zone plates" Microelectron. Eng. 35, 525-529 (1997). 